\title{
Les gens de mer du quartier de Marennes et l'institution des invalides de la Marine (milieu XVIIIe-milieu XIXe siècle)
}

Seafarers of the Marennes quarter and the institution of the "Invalides de la Marine" (mid-eighteenth to mid-nineteenth century)

\section{Thierry Sauzeau}

\section{(2) OpenEdition \\ Journals}

Édition électronique

URL : http://journals.openedition.org/abpo/2627

DOI : $10.4000 / a b p o .2627$

ISBN : 978-2-7535-2843-7

ISSN : 2108-6443

Éditeur

Presses universitaires de Rennes

Édition imprimée

Date de publication : 30 juin 2013

Pagination : 179-192

ISBN : 978-2-7535-2838-3

ISSN : 0399-0826

Référence électronique

Thierry Sauzeau, « Les gens de mer du quartier de Marennes et l'institution des invalides de la Marine (milieu XVIIIe-milieu XIXe siècle) », Annales de Bretagne et des Pays de I'Ouest [En ligne], 120-2 | 2013, mis en ligne le 30 juin 2015, consulté le 02 mai 2019. URL : http://journals.openedition.org/abpo/2627 ; DOI : 10.4000/abpo.2627 


\title{
Les gens de mer du quartier de Marennes et l'institution des invalides de la Marine (milieu XVIII ${ }^{\mathrm{e}}$-milieu XIX ${ }^{\mathrm{e}}$ siècle)
}

\author{
Thierry SAUZEAU \\ professeur d'histoire moderne, CRiHAM-EA4270 - Université de Poitiers
}

\begin{abstract}
Lorsqu'à la fin du XVII ${ }^{\mathrm{e}}$ siècle, Colbert mit sur pied le système des classes, il se proposait de mettre les gens de mer occupés à la pêche, au cabotage ou au long cours, sous la contrainte. Cette surveillance inédite sous l'Ancien Régime avait pour but la mobilisation - on disait alors la "levée " - de marins expérimentés pour constituer les équipages des navires de la flotte de guerre. D'abord mis en œuvre sous la seule contrainte, le système évolua vers une base contractuelle avec l'instauration d'avantages en échange du service. C'était tout simplement la naissance d'un nouveau privilège. Deux textes, l'un de 1681 et l'autre de 1689, mirent les arsenaux, la flotte et les équipages du roi en ordre de bataille avant d'affronter l'ennemi anglohollandais. En échange du service militaire, le titre IV du livre huitième de la grande ordonnance de la marine (1681) posait les bases de l'institution d'un secours, une "récompense des Officiers mariniers, matelots et soldats estropiez au service ${ }^{1}$ ". Il était question des " hospitaux que sa Majesté a destiné de faire bastir " mais aussi de la " demi solde ". Installé dans l'urgence d'un règne très belliqueux, le système ne put atteindre sa pleine maturité sous le règne de Louis XIV. Mises à mal par la Régence, les classes de la marine reçurent finalement, en la personne de Maurepas, un continuateur digne de leur fondateur. Ce ministre de Louis XV inaugura, avec le règlement du 10 mai 1728, une reconstruction étendue à l'institution des invalides. La notion de " hors de service " était alors reconnue de jure : un marin âgé de plus de 60 ans ne pourrait plus être appelé au service. En 1778, Sartine, ministre de Louis XVI, réaffirma les privilèges des gens de mer, avant que le maréchal de Castries ne régularise, grâce au règlement
\end{abstract}

1. "Ordonnance de Louis XIV pour les armées navales et les arcenaux de la marine (1689) ", publiée en pièce justificative dans MÉmain, René, Matelots et soldats des vaisseaux du Roi sous le règne de Louis XIV, Paris, 1937. 
du 31 octobre $1784^{2}$, une situation qui n'avait cessé d'évoluer tout au long du XVIII ${ }^{\mathrm{e}}$ siècle.

L'étude des profils des marins ayant pu bénéficier de l'exemption comme hors de service et/ou de la pension de demi-soldier (on dira aussi " pensionné ${ }^{3}$ ") dans le quartier de Marennes permet d'illustrer concrètement l'impact de cette institution sur le quotidien des gens de mer. $\mathrm{Au}$ nombre de 451, les hors de service recensés parmi les 3363 inscrits de la période 1760-1825 représentaient 13,4\% de l'effectif. Si l'on exclut les apprentis n'ayant pas poursuivi la navigation, cette cohorte rassemblait tout de même 19,4 \% des gens de mer du quartier. Loin d'être marginal, ce phénomène amenait un marin du quartier sur cinq à être considéré comme exempt du service et à pouvoir prétendre à une pension. Aussi convient-il de se pencher sur l'organisation de ces secours dispensés aux travailleurs de la mer, de l'Ancien Régime au XIX ${ }^{\mathrm{e}}$ siècle. Après une présentation des dispositifs en vigueur à la fin du XVIII ${ }^{\mathrm{e}}$ siècle, le profil des invalides devra ensuite être esquissé sans omettre d'examiner les états de service de chacun et les causes d'exemption ou d'indemnisation. Par la suite, on pourra vérifier comment les gens de mer invalides et hors de service assumaient leur quotidien : une étude de leurs activités sera alors esquissée et la fin de la vie de ces bénéficiaires de la protection sociale sera observée.

\section{Aux sources des systèmes de retraite par répartition : la caisse des invalides de la Marine}

La reconstitution des carrières des gens de mer des classes de Marennes en Saintonge permet de mesurer les effets d'une telle politique de solidarité. Possible sans lacune, à partir de 1760, l'étude des fins de carrières s'aligne principalement sur le texte de 1784 qui régla le passage aux hors de service et l'entretien, ou non, à la demi-solde jusqu'à la fin de l'Ancien Régime. En réalité, si les marins âgés de plus de 60 ans se voyaient classés hors de service, il y avait aussi les infirmes, accidentés du travail ou blessés de guerre, nécessairement plus jeunes. Pour tous, le dernier grade atteint au service - le classement - servait de base de calcul pour l'attribution de la demi-solde. Les hors de service pour " grand âge " étaient entretenus au prorata de leurs années de service. Pour les infirmes, le calcul était lié au degré d'incapacité et à l'origine de l'infirmité. L'invalide devait recevoir un tiers de sa dernière solde au service en cas d'incapacité partielle due à un accident survenu au commerce, une demi-solde en cas d'incapacité totale. Pour les accidents survenus au service, le taux allait de la demi-solde pour

2. CAPTIER, Jacques, Étude historique et économique sur l'inscription maritime, thèse de droit, Paris, 1907, p. 212.

3. Le terme est apparu dans la loi du 13 mai 1791; il fut d'abord réservé aux inscrits dont la solde était supérieure ou égale à 81 francs au service (grade de chef timonier au service) puis il a été généralisé au XIXe siècle. Voir Lemagnen, Sylvette, Aspects de la vie maritime dans le Quartier de Cherbourg (1816-1850), univ. Caen, mémoire de maîtrise, 1983, p. 106. 
incapacité partielle aux deux tiers pour une incapacité totale. Depuis le règne de Louis XIV, une comptabilité commune unissait la caisse des invalides (cotisation salariale de 2,5\% pour les marins au commerce), la caisse des gens de mer (reliquat de soldes pour désertion, insubordination, décès sans héritier) et la caisse des prises (activité des corsaires); elle permettait à la Trésorerie générale d'enregistrer des excédents. Par ailleurs, une compensation géographique liait les trésoreries particulières des quartiers. Les excédents des grands ports, riches d'abondantes cotisations, étaient ventilés vers les quartiers ruraux afin de subvenir aux besoins des gens de mer. Le règlement des invalides de janvier $1786^{4}$ précisait les conditions d'accès à la demi-solde et de versement de celle-ci. L'article II stipulait qu'" aucun Invalide ne [serait] porté sur les matricules que sur présentation de l'ordre du Roi, ou de l'extrait des listes signées en commandement ". L'article I réglait que "les invalides [seraient] payés, à l'avenir, lors des revues qui [seraient] faites à la révolution précise de chaque semestre ». Sur ces bases, les textes révolutionnaires vinrent remodeler l'institution des invalides. La " loi sur les classes des gens de mer " (7 janvier 1791) abaissa d'emblée à 56 ans l'âge de " dispense de l'obligation au service ". Remplaçant les classes de la marine par l'inscription maritime, la loi du 3 brumaire an IV (25 octobre 1795) porta même cet âge à 50 ans.

Par la suite, les lois sur l'inscription maritime ${ }^{5}$ n'ont plus remis en cause cet âge. Seuls les modes de calcul des services et de l'indemnité auxquels ils ouvraient droit continuèrent à faire débat. En effet, si l'Ancien Régime finissant avait laissé à la discrétion du pouvoir royal l'inscription sur la liste des demi-soldiers ${ }^{6}$, la Révolution donna le coup d'envoi d'une période de rationalisation et de normalisation. Il n'entre pas dans le cadre de cette étude de traiter de l'évolution des modes de calcul des droits à la demisolde; il faut cependant remarquer que progressivement, l'administration en vint à prendre en compte, au-delà du service, les années de navigation au commerce puis à la petite pêche. Ainsi, pour l'attribution de la demi-solde, le système des classes ne tenait compte que des seuls services dans la marine royale. À partir du 3 brumaire an IV, l'inscription maritime comptabilisa les navigations commerciales. Une année au long cours ou au cabotage comptait désormais pour 6 mois, un an au service pour 18 mois. Enfin, sous la Restauration, les ordonnances des 17 septembre 1823 et 24 janvier 1824 ouvrirent la prise en compte du temps passé à la pêche ou au pilotage ${ }^{7}$, sur

4. Service Historique de la Défense, Rochefort, sous-série $2 \mathrm{P}$ « service de Classes puis de l'Inscription Maritime ", 2P5 "Instructions relatives aux fonds des Invalides et des Gens de Mer, janvier 1786 ".

5. Évoqués par CAPTIER, Jacques, Étude historique et économique... op. cit.; les textes figurent dans BLANCHARD, René, Répertoire général des lois, décrets, ordonnances, règlements et instructions sur la Marine, Paris, Impr. Nat., 1848-1850, 2 vol.

6. Certains ont pu être retrouvés sur les listes de "Proposition à la demi-solde ", Archives Nationales, fond Marine, C 4.

7. La prise en compte du pilotage devait enrayer la désaffection des jeunes pour ce métier, Lemagnen, Sylvette, Aspects de la vie maritime dans le Quartier de Cherbourg..., op. cit., p. 22. 
la base de 6 mois de service pour 1 an de navigation. Au total, l'institution des invalides de la marine n'a cessé de se développer et de préciser ses règles de fonctionnement.

\section{La réalité des secours distribués aux gens de mer de l'estuaire de la Seudre}

La biographie collective de plusieurs centaines de gens de mer entrés dans la carrière entre 1760 et 1825 entraîne un certain décalage chronologique lorsqu'il s'agit d'étudier leur profil de retraité. Âgé d'une quinzaine d'années en 1760, un mousse atteignait l'âge légal de la retraite d'Ancien Régime - 60 ans - en 1805. Or, à cette époque, l'inscription maritime avait organisé un nouveau régime qui faisait passer l'inscrit hors de service à 50 ans. Il n'en demeure pas moins qu'on recense 157 hors de service - 34,8 \% - âgés de moins de 50 ans à leur passage aux invalides. Les accidents du travail et blessures de guerre étaient donc responsables du tiers des exemptions de service, même s'ils ne débouchaient pas systématiquement sur le versement d'une pension. Au sein de la population d'ayants droit, l'évolution historique du rapport entre retraités et infirmes éclaire finalement les mutations d'une politique sociale au sein de laquelle, progressivement, la fonction de récompense prend le pas sur celle de la réparation.

Figure 1 - Structure par âge des invalides

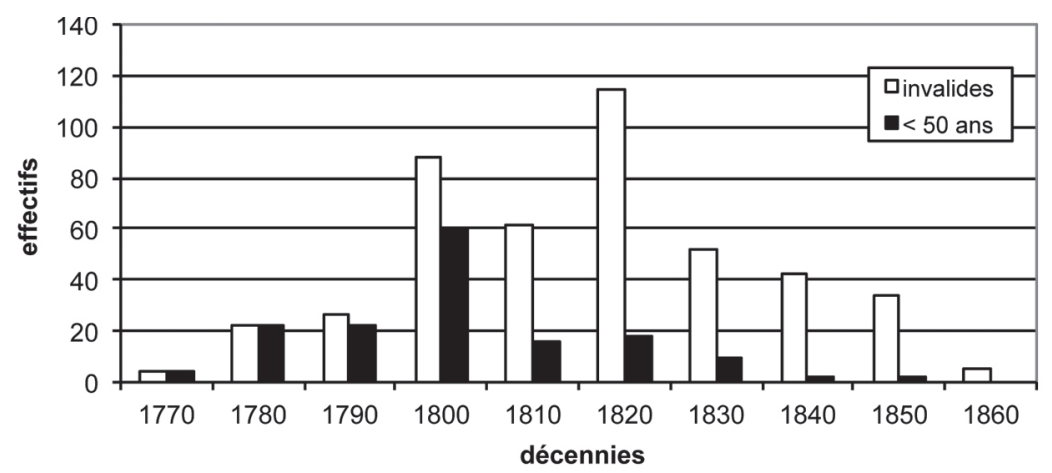

Au cours des seules décennies 1770 à 1800, les hors de service et invalides de la cohorte étudiée affichaient ces mêmes moyennes d'âge précoce : les classés hors de service l'étaient en grande majorité sur blessure ou accident du travail. Les dernières décennies d'Ancien Régime avec 30 " passés aux hors de service ", dont 13 étaient admis à la demi-solde, illustrent pourtant bien la politique volontariste d'un Louis XVI soucieux des choses de la mer, attentif aux gens de mer et épaulé par des ministres 
compétents. Charles Deloumeau ${ }^{8}$ (Bourcefranc, né en 1751, inscrit de 1762 à 1787) " estropié sur le vaisseau le Triton " et débarqué à Cherbourg par le Sieur d'Estienne fut ainsi admis aux hors de service et à la demi-solde " par ordre du Roi ". Même si les demi-soldes étaient irrégulièrement versées, la monarchie absolue semble les avoir plus largement distribuées, alors que leur attribution était restée limitée durant le XVIII ${ }^{\mathrm{e}}$ siècle ${ }^{9}$. Les décennies 1790 et 1800 vinrent rompre avec cette politique d'indemnisation des " estropiés " faute de crédits, les ayants droits s'étant multipliés par suite de la guerre.

\section{Figure 2 - Invalides du quartier de Marennes (cohorte des inscrits 1760-1825)}

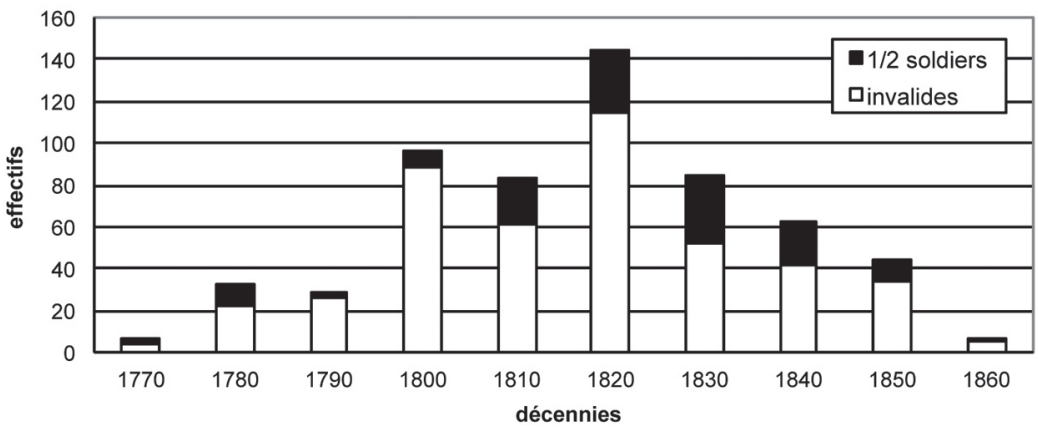

Sans argent mais avec un cruel besoin de main-d'œuvre, la République choisit de maintenir le système d'exemption du service mais limita ses secours à quelques situations désespérées : 8 sur 114 . Pierre Graslé ${ }^{10}$ ('Éguille, né en 1753, inscrit de 1766 à 1795), maître d'équipage sur la frégate L'Expériment était ainsi apostillé " a eu le bras coupé durant la campagne "; le $1^{\text {er }}$ octobre 1795, il fut admis aux invalides. Plusieurs facteurs expliquaient sans doute le bon rapport d'indemnisation observable à partir de 1810. Démobilisée sous l'Empire, la marine était une arme bien moins dangereuse que le service par terre, tandis que les activités commerciales et de pêche demeuraient côtières : les marins estropiés cédèrent la place

8. Service Historique de la Défense, Rochefort, début de carrière sur le Registre des Mousses du quartier de Marennes (1764-1776) 10P3.1, f ${ }^{\circ} 56, \mathrm{n}^{\circ} 54$.

9. Comptages réalisés par BOYER, Grégory, La Marine berckoise de Louis XIV à 1945 : vie et mort d'un port de pêche, univ. du Littoral, thèse de doctorat en histoire, 2001. Ils donnent ainsi 86 matelots passés aux Invalides entre 1706 et 1789, mais seulement 8 admis à la demie solde, p. 67.

10. Service Historique de la Défense, Rochefort, début de carrière sur le Registre des Mousses du quartier de Marennes (1776-1790), 10P3.3, $\mathrm{f}^{\circ} 154, \mathrm{n}^{\circ} 9$. 
aux " hors d'âge ". Enfin, le rétablissement des finances publiques permit assurément une meilleure indemnisation ${ }^{11}$.

Cela n'empêchait nullement les retards de classement aux invalides, une habitude permanente depuis le XVIII ${ }^{\mathrm{e}}$ siècle ${ }^{12}$. Étienne Bertrand ${ }^{13}$ (Marennes, né en 1777, inscrit de 1800 à 1844) fut ainsi " classé hors de service d'office au $3^{\mathrm{e}}$ trimestre 1843 " alors qu'il était entré dans sa $66^{\mathrm{e}}$ année! Encore n'était-il pas question, pour lui, de revendiquer un quelconque droit à la demi-solde... Techniquement, le classement dans les hors de service dépendait de deux types de facteurs, l'un structurel, l'autre conjoncturel. De la tenue "à jour " des registres du quartier découlait le recensement par le sous-commissaire aux classes des individus susceptibles d'être " passés aux H.S. ». Point noir, une interrogation de notre base de données fait apparaître la présence de 109 marins âgés de plus 50 ans à la fin de leur carrière mais n'ayant pas fait l'objet d'un classement aux H.S. Abus d'autorité de la part d'une marine à la recherche de matelots pour la flotte? Volonté des inscrits quinquagénaires de ne pas se rappeler au bon souvenir de l'inscription maritime dans des périodes troublées? C'est sous l'Empire et la Restauration que le phénomène a connu ses maxima. Au moment de leur cinquantième anniversaire, le statut des individus concernés fait apparaître tout un lot de situations ayant entraîné une amnésie administrative plus ou moins structurelle. L'inactivité prolongée de 25 des oubliés était à l'origine de leur absence des listes de H.S. On recense d'autre part 29 marins officiellement maintenus "sous les drapeaux " (13 au service, 8 en captivité et 8 employés aux passages d'eau ou aux tours de guet), que l'administration avait vraisemblablement oublié de rayer des actifs. Plus loin, 19 marins, patrons ou matelots des barques n'avaient peut-être pas jugé utile de se faire déclasser, à moins qu'en période de crise de l'État, l'administration n'ait montré que peu d'empressement à grossir les rangs des candidats à la demi-solde... Restés outre-mer sans avoir été considérés comme déserteurs, 8 quinquagénaires étaient loin du quartier. On comptait encore 8 gens de mer apparus sur les matricules après leurs 50 ans! Enfin, 15 titulaires de congés illimités ou définitifs - souvent pour cause de blessure - continuaient d'être classés aux matelots alors même qu'ils étaient de facto hors de service. Le passage aux hors de service n'était donc pas toujours accordé, souvent par négligence de l'administration et par ignorance des intéressés.

11. Des comptages réalisés par sondages chronologiques dans les matricules ont permis d'enregistrer le même phénomène et de le mesurer. Dans le Calvados, Dominique BRIAND a compté $6 \%$ de demi soldiers parmi les H.S. en 1817, mais $31 \%$ en 1845 et $38 \%$ en 1857 (Hommes et navires de la région de Caen-Courseulles (1816-1861), univ. Caen, mémoire de maîtrise, 1983, p. 115).

12. Grégory BOYER cite le cas de Claude Dez (Service Historique de la Défense, Cherbourg 2P2.21) inscrit à la demie solde en juin 1754, alors qu'il est décédé depuis avril, ce qui illustre bien le fait que les délais de classement étaient parfois très longs (La marine berckoise de Louis XIV à 1945..., op. cit.).

13. Service Historique de la Défense, Rochefort, début de carrière sur le Registre des Novices du quartier de Marennes (an XII-1815), 10P3.20, $\mathrm{f}^{\circ} 22, \mathrm{n}^{\circ} 243$. 
De manière plus conjoncturelle, la décision de classement était souvent retenue à l'échelon du quartier et ne se débloquait qu'à la faveur d'un événement exceptionnel. Cela pouvait venir d'une revue d'effectif dirigée par un inspecteur. En octobre-novembre 1803, le général Cambis, nommé par Napoléon à la tête de la réforme des Équipages permanents, présida une revue dans le quartier et procéda au classement de 47 individus aux H.S. En juillet 1810, l'inspecteur des classes Dupéreau distinguait à son tour 15 marins. L'habitude perdura après 1815. Par exemple, l'inspecteur Gachot exempta 31 marins durant la seule journée du 20 septembre 1816, le contre-amiral Baudin fit de même avec 20 inscrits le $1^{\mathrm{er}}$ avril 1823. À côté des revues d'effectif, les levées donnaient aux services de santé l'occasion d'exempter nombre d'individus. En septembre 1789, le chirurgien de la marine Vénot déclarait H.S. 6 marins et, en mars 1793, le chirurgien major de la marine Clémot en "réformait " 6 autres. C'est un officier de santé qui procédait à la revue du $1^{\text {er }}$ floréal an V (20 avril 1797), au sortir de la Terreur, il attribuait à 8 marins un " bulletin d'exemption définitive». Le classement aux H.S. était donc la conséquence d'une décision de l'autorité centrale, sanitaire durant la Révolution, et de plus en plus administrative ensuite. Il faut noter qu'après la visite du contre-amiral Baudin, les classements aux H.S. se firent plus rigoureux et systématiques : l'individu était alors dit " porté aux H.S. sur décision de Son Excellence le Ministre de la Marine ". Avec la normalisation des critères d'accès aux H.S., les gens de mer finirent donc par être traités à égalité. Il n'empêche que l'examen des derniers classements des H.S. donne un avantage numérique aux " gradés " (52\%) sur les simples matelots. Nulle discrimination dans ce rapport mais plutôt l'illustration nette d'une situation sociale incertaine pour les matelots, plus exposés aux risques de la navigation, en temps de paix comme en temps de guerre. Il en résultait une espérance de vie moindre et la disparition de nombreux " prolétaires de la voile " bien avant l'âge légal de la retraite.

La discrimination était ailleurs, dans l'octroi de la demi-solde à 179 individus (39,7 \% des H.S.) notamment. Un matelot H.S. sur six environ touchait finalement une pension. Du côté de ceux qui s'étaient distingués au service, comme gabier ou par un "grade " d'officier marinier, la proportion s'élevait à la moitié. Ainsi, les résultats des savants décomptes de la caisse des invalides étaient-ils en conformité avec ceux du tableau d'avancement militaire, sanctionné par le classement. La prime au patriotisme était évidente dans la mesure où les services de l'État étaient affectés d'un coefficient 1,5 dans les décomptes qui n'associaient aux navigations civiles (commerce, pêche et pilotage) qu'un coefficient 0,5. Étudié dans le quartier d'Auray, à la fin du $\mathrm{XIX}^{\mathrm{e}}$ siècle, le même phénomène touchait les matelots H.S., dont seulement $21 \%$ percevaient la demi-solde ${ }^{14}$.

14. Aulard, Patricia, Mobilité et adaptation chez les inscrits maritimes des Quartiers de Vannes et Auray dans la $2^{\text {nde }}$ moitié du XIX siècle, univ. Rennes II, mémoire de DEA, 1995, présente ces résultats en se basant sur le registre 6P2.27 des Hors de Service et Pensionnés du Quartier d'Auray (1876-1883). Assortie d'un récapitulatif des services, 
Figure 3 - Classement d'origine des invalides

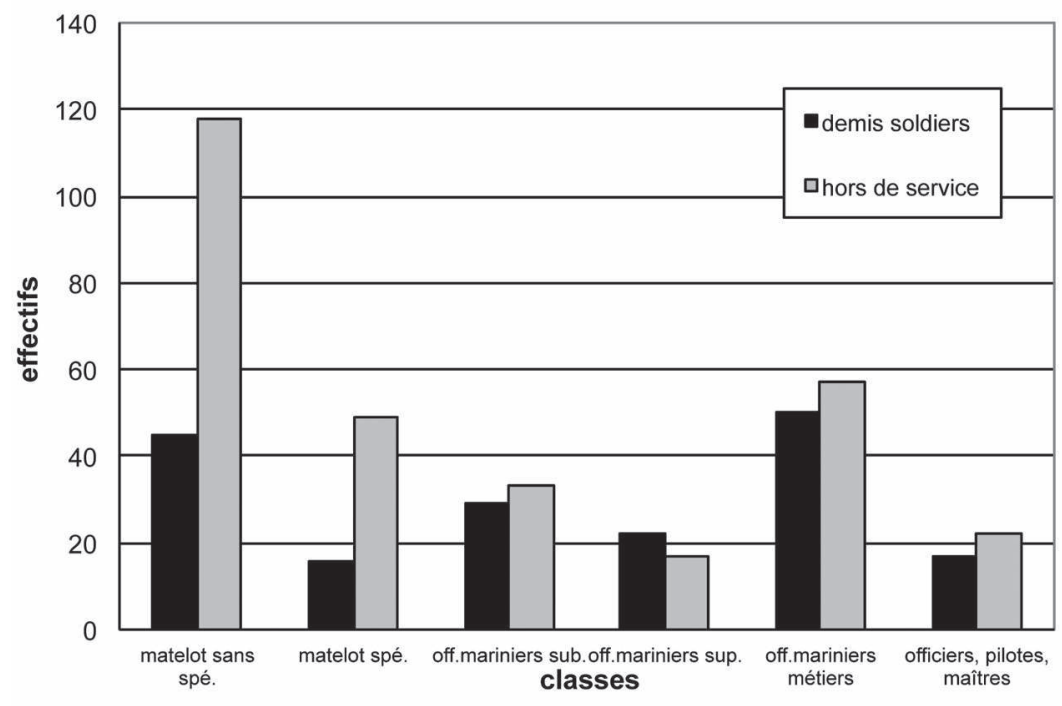

Deux marins de l'Éguille peuvent servir à illustrer la barrière séparant H.S. et demi-soldiers. Clément Chemin ${ }^{15}$ (L'Éguille, né en 1810, inscrit de 1819 à 1865) et Laga Chardavoine ${ }^{16}$ (L'Éguille, né en 1810, inscrit de 1820 à 1865) sont deux contemporains, nés la même année, dans deux familles de pêcheurs d'estuaire. Ils ont débuté leur carrière comme mousses, très jeunes, comme l'autorisait l'apprentissage sur la barque familiale et dans les eaux calmes de la Seudre et comme le commandait le souci de leur éviter le service dans la Grande Armée, en faisant d'eux, au plus tôt, de vrais et incontestables inscrits maritimes. Ils sont ensuite passés matelots, le premier dans sa $19^{\mathrm{e}}$ année, le second dans sa $20^{\mathrm{e}}$. Patrons de barque vers 27 ans pour Clément, vers 34 ans pour Laga, ils passèrent tous les deux H.S. par décision ministérielle, début décembre 1860, dans leur $50^{\mathrm{e}}$ année. Semblables, leurs carrières ne débouchèrent cependant pas sur un même droit à une pension. Tout en ayant travaillé davantage - enrôlé 91,6 \% du temps - que Laga Chardavoine (85,5\%), Clément Chemin ne fut pas admis à la demi-solde. À y regarder de plus près, ce qui fit pencher la balance du côté du moins actif, ce fut la durée passée au service (77 mois contre 69). Affecté du coefficient 1,5 pour le calcul des pensions (contre 0,5 pour les autres navigations), le service crédita Laga Chardavoine de 325 mois alors

cette matricule permet de séparer les H.S. en trois catégories : les demi-soldiers, les H.S. qui naviguent en vue de compléter leurs services, et une catégorie apostillée "n'auront jamais le temps", p. 173.

15. Service Historique de la Défense, Rochefort, début de carrière sur le Registre des Mousses du quartier de Marennes (1815-1825) 10P3.27, $\mathrm{f}^{\circ} 13, \mathrm{n}^{\circ} 102$.

16. Idem, $10 \mathrm{P} 3.27, \mathrm{f}^{\circ} 15, \mathrm{n}^{\circ} 119$. 
que Clément Chemin n'afficha que 299 mois. Résultat? Laga fut admis à la demi-solde le 5 mars 1861 tandis que Clément n'y eut pas droit. Sous le second Empire, le seuil d'admission aux demi-soldiers tournait donc autour des 300 mois - comptables - de service, sans possibilité de faire prendre en compte les navigations ultérieures ${ }^{17}$. La gestion du dossier des invalides à Marennes était-elle représentative de la mise en œuvre de la politique sociale de la marine aux différentes époques? Il semble qu'un peu plus de $80 \%$ des ayants droit ${ }^{18}$ étaient versés aux H.S., les autres devenant H.S. de facto, sans faire l'objet d'une inscription particulière. Parmi les H.S. de jure, un peu moins de $40 \%$ de demi-soldiers devaient finalement être inscrits sur les listes de pensionnés, dans un apparent respect des critères de service. Cependant, comme partout sur le littoral ${ }^{19}$, la demi-solde était irrégulièrement versée et ce problème occupa la majeure partie de la correspondance au départ du bureau de Marennes ${ }^{20}$. Les opérations de versement de 1797 donnent une idée des difficultés en cas de crise : le 3 prairial an V (22 mai 1797), le sous-commissaire Josse informa les syndics de La Tremblade, Chaillevette et Saujon que la demi-solde du premier semestre était à la disposition des invalides au bureau. Cependant, le 28 novembre, Josse alerta l'ordonnateur de la marine ${ }^{21}$ que « les 4000 livres [sic] envoyées [étaient] insuffisantes pour payer les demi-soldes promises aux invalides ". Il semble que l'indemnisation du second semestre ait été plus difficile à honorer, puisqu'une nouvelle requête fut envoyée à Rochefort en ce sens, le 28 décembre... Cet exemple suffit à démontrer que les secours étaient trop faibles et irréguliers pour faire subsister l'invalide et sa famille.

\section{Faiblesse des pensions, poursuite de la navigation}

Pour 227 marins "versés " aux H.S. - 50,3 \% des retraités/infirmes -, la poursuite d'une activité professionnelle était finalement inévitable. Les registres matricules du quartier révèlent 1678 enrôlements réalisés par ces retraités, soit 7,4 par marin. La structure des activités laisse la part belle aux petites navigations ( $96 \%$ ), même si quelques individus ne renonçaient pas au long cours (12 marins) ou au service " de bonne volonté " (18). Limitées aux mers bordières de la Seudre, les activités l'étaient aussi dans le temps puisque plus de la moitié des H.S. ne réalisaient pas plus de 5 campagnes de pêche ou transport tandis que les H.S. ayant réalisé de 6 à

17. Ce que la III République semble avoir autorisé suivant Patricia Aulard (Mobilité et adaptation chez les inscrits maritimes des Quartiers de Vannes et Auray... op. cit., p. 173).

18. On entend par cette expression les " estropiés " et autres " hors d'âge ", âgés de plus de 50 ans aux termes des règlements postérieurs à la loi du 3 brumaire an IV ( 25 octobre 1795).

19. Grégory Boyer indique que " cette demi-solde pouvait parfois mettre des années avant de parvenir aux matelots " (La Marine berckoise de Louis XIV à 1945... op. cit., p. 67).

20. Service Historique de la Défense, Rochefort. Correspondance au départ du bureau de l'Inscription Maritime de Marennes, 10P2.1 (pluviose an V à thermidor an VIII) et 10P2.2 (thermidor an VIII à 1810).

21. Successeur révolutionnaire de l'intendant de marine avant l'installation d'un préfet. 
10 voyages constituaient $25 \%$ de l'effectif. Seul un petit quart des retraités avaient donc une activité prolongée. Un créneau existait sur le marché pour ces invalides, en rapport avec leurs compétences, puisque 74,8\% des enrôlements l'étaient comme patron de barque. Cette tendance était d'ailleurs accentuée chez les titulaires de plus de 10 campagnes qui étaient patrons sur 90,3 \% des rôles. Avec une moyenne d'âge à l'entrée établie à 48 ans et une disparition des registres à l'âge moyen de 62 ans, c'étaient en fait 14 années dont disposait, statistiquement, chaque invalide pour continuer à naviguer. Cette moyenne recouvrait bien une réalité pour 50,6\% des invalides, ceux qui étaient entrés à 49 ou 50 ans aux hors de service et qui avaient pu poursuivre leur carrière entre 10 et 20 ans. Versés aux H.S. à un âge moyen ne dépassant pas 47 ans, pour l'autre moitié, les invalides, les plus jeunes, adoptaient des profils diamétralement opposés.

Figure 4 - Navigations des H.S.

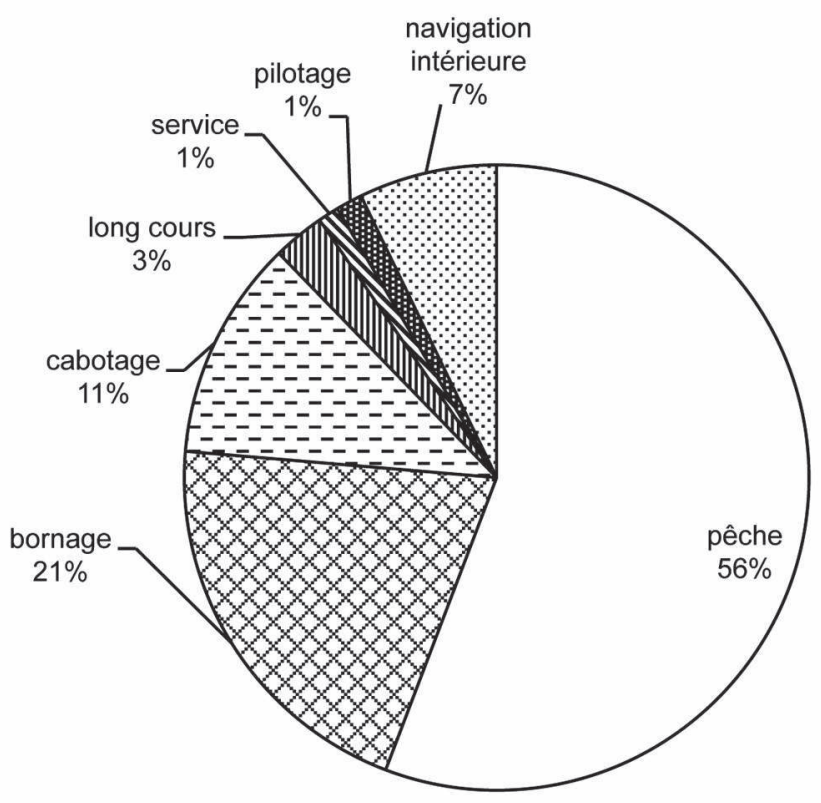

Pour 28,5 \% l'expérience ne se poursuivait pas au-delà de la cinquième année, tandis qu'à l'inverse 20,9 \% prolongeaient leurs activités au-delà de la $20^{\mathrm{e}}$ année. Versés aux invalides pour raisons médicales, les sujets les plus jeunes qui survivaient à leur pathologie avaient une espérance de vie prolongée : leur disparition des matricules intervenait à 73 ans en 
moyenne. Pierre Hervé ${ }^{22}$ (Chaillevette, né en 1747, inscrit de 1769 à 1775) fut versé aux H.S. le $1^{\mathrm{er}}$ mai 1775, avec l'apostille « hors d'état de naviguer et de gagner sa vie, a les jambes perdues ". Dans son cas, le pronostic était tristement exact et sa jeunesse (28 ans) n'empêchait pas une inactivité immédiate et définitive. À l'inverse, H.S. à 28 ans lui aussi, Jean Baptiste Tuloup $^{23}$ (Marennes, né en 1776, inscrit de 1788 à 1834) malgré l'apostille du port de Toulon, "pied droit écrasé et par suite des dépôts à la jambe, gêne des mouvements ", navigua de 1803 à 1834 à la petite pêche et au bornage. La condition d'invalide, pensionné ou non, était donc très variable en fonction des circonstances de passage aux hors de service. Si tous les marins exempts du service étaient placés devant l'impératif économique de poursuivre la navigation pour gagner de quoi subsister, tous n'étaient pas en état de le faire. La moitié des invalides, les exemptés car " hors d'âge ", avaient encore quelques forces et un savoir-faire à mettre au service des barques du quartier. Les plus jeunes, versés aux H.S. sur blessure ou maladie, ne purent envisager de poursuivre leur activité professionnelle qu'au terme d'une convalescence réussie. Sur cinq estropiés, seuls deux pouvaient, avec le temps, reprendre une activité professionnelle comme matelot ou patron de barque et la prolonger aussi longtemps que leur âge le leur permettait. La majeure partie des réformés n'était cependant plus apte à aucun type de navigation et ces hommes quittaient aussitôt le métier. Eu égard à la faiblesse de leurs services et au caractère mal défini de leur pathologie, ils recevaient le secours d'une demi-solde dans un tiers des cas seulement.

Le bureau des classes de Marennes offre donc un exemple de traitement de la question des invalides entre la fin du XVIII et le XIX ${ }^{\mathrm{e}}$ siècle. Avec $20 \%$ de gens de mer classés, finalement versés aux hors de service (451 sur 2325 ), la Saintonge ne se démarquait pas de la Manche orientale (Cherbourg, Caen, Berck). La croissance de la proportion de demi-soldiers parmi les H.S. (39,7 \%) était tout aussi comparable aux quartiers de référence : $10 \%$ sous l'Ancien Régime jusqu'à $50 \%$ au milieu du XIX ${ }^{\mathrm{e}}$ siècle. Cependant, le recours à la prosopographie permet de faire apparaître un groupe de marins - 109 " hors d'âge " n'ayant été versés ni aux H.S. ni à la demi-solde. Parmi les gens de mer âgés, on comptait finalement un " exclu " pour quatre H.S. Sans établir clairement les responsabilités de cette omission, il convient finalement d'en relativiser la portée : ces marins profitaient de facto d'une situation d'exemption. Au reste, tous les hors de service, y compris ceux qui percevaient - avec difficulté - la demi-solde, devaient poursuivre une activité autant que leur santé le leur permettait. Tous n'étaient d'ailleurs pas des marins " hors d'âge " puisque de la cohorte 1760-1825, sortirent 157 " estropiés " et autres " malades " âgés de moins de 50 ans. La période

22. Service Historique de la Défense, Rochefort, début de carrière sur le Registre des Novices du quartier de Marennes (1764-1790), 10P3.4, $\mathrm{f}^{\circ} 156, \mathrm{n}^{\circ} 26$.

23. Service Historique de la Défense, Rochefort, début de carrière sur le Registre des Officiers mariniers et matelots du quartier de Marennes (1796-an XII), 10P3.18, $\mathrm{f}^{\circ} 41, \mathrm{n}^{\circ} 60$. 
révolutionnaire et l'Empire virent se multiplier ces incidents de parcours. Le diagnostic médical et son pronostic étaient assez sûrs pour que trois exemptés sur cinq disparaissent du métier, incapables de naviguer davantage. Centrée sur les barques du quartier, une retraite active attendait les rescapés : c'est en septuagénaires qu'ils mettaient finalement sac à terre. Elle était aussi le lot commun des inscrits hors d'âge, versés aux H.S. La petite pêche et les navigations à très faible rayon d'action (bornage, navigation intérieure) constituaient l'essentiel de leurs activités. Sur le rôle d'armement, l'inscrit H.S. était embarqué comme patron trois fois sur quatre. Ce recours systématique à la navigation mettait finalement en lumière les limites d'un système de protection sociale unique en son genre dans la société d'Ancien Régime et du premier XIX ${ }^{\mathrm{e}}$ siècle. Plus qu'un droit au repos après des années de labeur, la demi-solde était une sorte de gratification patriotique, dans la mesure où elle s'adressait en priorité aux individus ayant servi l'État. Pour la minorité qui touchait cette demi-solde, ce revenu minimum était insuffisant et nécessitait la poursuite d'une activité normale. Exemple de privilège maintenu et développé au-delà de l'Ancien Régime, l'institution des invalides de la marine a finalement permis aux membres les plus âgés de la population maritime de subsister, en leur donnant les moyens de poursuivre leurs activités au milieu des gens de mer classés. Elle était exemplaire d'une modernité réelle de l'institution maritime. Elle a inauguré les pratiques de répartition ou de solidarité entre caisses, entre régions, finalement entre générations, toutes choses constitutives d'une citoyenneté sociale adossée à un État Providence que l'on croirait à tort inventé au Xx siècle.

Relevant du privilège d'Ancien Régime, le statut d'invalide de la marine a évolué au gré des régimes politiques. La loi instituant l'inscription maritime ( 3 brumaire an IV) sur des critères de plus en plus objectifs et comptables abolit bien le fait du prince pour l'attribution des exemptions de service et la demi-solde. Si 451 inscrits marennais purent finalement bénéficier de l'exemption de service, seuls 179 d'entre eux bénéficièrent de l'octroi d'une demi-solde pour retraite. Pour les classes du quartier de Marennes, dont le centre de gravité se faisait plus populaire au XIX ${ }^{\mathrm{e}}$ siècle, le bilan de la politique sociale de la marine apparaît cependant contrasté. En effet, seuls $20 \%$ des gens de mer classés accédèrent aux hors de service et parmi eux, pas plus de 40 \% finirent pensionnés. Seule la poursuite d'une activité professionnelle pouvait permettre aux invalides de subsister. Issus de la bourgeoisie ou des couches populaires de la société maritime, les gens de mer du quartier ont donc pris part à la gestation d'une institution contemporaine, née sous la monarchie absolue. La prosopographie d'un petit peuple de marins ruraux en illustre quelques caractères. Le destin des gens de mer inscrits entre 1760 et 1825 à Marennes est significatif d'une évolution vers un système de protection sociale contemporain. Des progrès ont été enregistrés dans le respect de règles de plus en plus transparentes pour le pas- 
sage aux hors de service. Simultanément, le pourcentage de marins admis à la demi-solde ne cessa de croître, préfigurant un souci de protection sociale croissant des autorités ${ }^{24}$. Dans le quartier de Marennes, l'invalide semble avoir préfiguré les premiers développements d'un embryon de citoyenneté sociale. Ainsi, au début du Second Empire, les promesses sociales de l'Ancien Régime paraissent enfin tenues. Suit immédiatement une période de turbulences, lorsque Napoléon III fait basculer la France dans une ère libérale et industrielle. À bord, la motorisation de la marine marchande et la professionnalisation de la marine de guerre entraînent une dévalorisation du personnel issu de l'inscription maritime, matelots des navigations et pêches côtières en majorité. Devenus moins nécessaires à l'économie de la défense nationale, ces gens de mer se retrouvent accusés de profiter du système des invalides, sans assumer les charges du service militaire telles que les avait définies Colbert. Pourtant tant décriés, ces modestes acteurs sont à l'origine du rebond de l'économie maritime à Marennes, avec le développement de l'ostréiculture dont la valeur ajoutée redonna un nouveau souffle à l'institution des invalides, au Xx ${ }^{\mathrm{e}}$ siècle ${ }^{25}$.

24. Le mouvement mutualiste est impulsé par un décret de Louis Napoléon en 1852 (TOUCAS, Patricia, "Solidarité mutualiste et prévoyance maritime sur le littoral charentais aux XIX ${ }^{\mathrm{e}}$ et XX ${ }^{\mathrm{e}}$ siècles ", dans Actes de la Table ronde du Centre de Recherche sur les Sociétés Littorales du Ponant, Lorient, janv. 1995).

25. À Marennes, on peut ainsi reconnaître la validité de l'analyse menée en Bretagnesud autour des économies sardinière et ostréicole par LE BoUËDEC, Gérard, LE HUNSEC, Mathieu et MALONDA, Frédéric, "Les inscrits à la petite pêche et leur rapport au service de la Marine : l'exemple de la Bretagne-sud au XIX ${ }^{\mathrm{e}}$ siècle ", dans LE BouËDEC, Gérard (dir.), Histoire du cabotage européen aux XVte et XIXe siècles, PUPS, Revue d'Histoire Maritime, $\mathrm{n}^{\circ} 8$, 2008, p. 187-205. 


\section{RÉSUMÉ}

Pendant le règne de Louis XIV, la monarchie française essaie de mettre en place un nouveau système de recrutement des marins de la flotte de guerre : le système des classes. Les marins résistent à ce projet et Colbert doit leur offrir des contreparties. Parmi elles, figure l'institution des invalides, pour les marins âgés ou blessés. Alimentée par des cotisations sur les salaires des pêcheurs et des matelots du commerce, une caisse organise les secours pour les invalides. En suivant les générations de marins inscrits dans le quartier de Marennes (France, Charente-maritime) entre 1760 et 1825, cette communication montre qu'il a fallu attendre plus d'un siècle pour voir cette organisation fonctionner correctement, à la veille de la Révolution. Au XIx ${ }^{\mathrm{e}}$ siècle, la croissance des pêches et des navigations côtières permet l'attribution de secours à ceux qui en ont besoin, avant une remise en question à la fin du siècle.

\section{ABSTRACT}

During the reign of Louis XIV, the French monarchy tried to implement a new system of recruitment for seamen of the fleet: the class system. Sailors opposed this project and Colbert was forced to offer some concessions in return. Among them, was the institution of the "invalides", for old or injured sailors. Underwritten by contributions taken from the wages of fishermen and sailors engaging in trade, the fund organised support for the retired seamen. By documenting the generations of sailors registered in the district of Marennes (France, Charente-Maritime) between 1760 and 1825, this paper shows that it took over a century for this organisation to be run correctly and was effective only shortly before the Revolution. In the nineteenth century, the growth of fisheries and coastal navigation allowed relief to be granted to those in need, though this was increasingly challenged by the end of the century. 